\title{
A Parking Function Setting for Nabla Images of Schur Functions
}

\author{
Yeonkyung Kim
}

Mathematics Department, University of California, San Diego, La Jolla, CA, USA

\begin{abstract}
In this article, we show how the compositional refinement of the "Shuffle Conjecture" due to Jim Haglund, Jennifer Morse, and Mike Zabrocki can be used to express the image of a Schur function under the Bergeron-Garsia Nabla operator as a weighted sum of a suitable collection of "Parking Functions." The validity of these expressions is, of course, going to be conjectural until the compositional refinement of the Shuffle Conjecture is established.

Résumé. Dans cet article, nous montrons comment le raffinement compositionel de la "Conjecture Shuffle" due à Jim Haglund, Jennifer Morse et Mike Zabrocki peut être utilisé pour exprimer l'image d'une fonction de Schur sous l'opérateur Nabla de Bergeron-Garsia comme une somme pondérée d'un ensemble convenable de "fonctions parking." La validité de ces expressions, bien sûr, va être conjecturale jusqu'à ce que le raffinement de la composition de la "Conjecture Shuffle" est établie.
\end{abstract}

Keywords: Parking Function, Nabla, Hall-Littlewood operators

\section{Introduction}

Parking Functions in the $n \times n$ lattice square are represented in the computer by two line arrays

$$
P F=\left[\begin{array}{llll}
v_{1} & v_{2} & \cdots & v_{n} \\
u_{1} & u_{2} & \cdots & u_{n}
\end{array}\right]
$$

with $u_{1}, u_{2}, \ldots, u_{n}$ integers satisfying

$$
u_{1}=0 \quad \text { and } \quad 0 \leq u_{i} \leq u_{i-1}+1
$$

and $V=\left(v_{1}, v_{2}, \ldots, v_{n}\right)$ a permutation in the symmetric group $S_{n}$ satisfying

$$
u_{i}=u_{i-1}+1 \Longrightarrow v_{i}>v_{i-1} .
$$

We will denote by $\sigma(P F)$ the permutation obtained by successive right to left readings of the components of the vector $V=\left(v_{1}, v_{2}, \ldots, v_{n}\right)$ according to decreasing values of $u_{1}, u_{2}, \ldots, u_{n}$. We will call $\sigma(P F)$ the "diagonal word" of $P F$. We will also let $i \operatorname{des}(P F)$ denote the descent set of the inverse of $\sigma(P F)$.

\footnotetext{
${ }^{\dagger}$ work accomplished with NSF support

1365-8050 @ 2013 Discrete Mathematics and Theoretical Computer Science (DMTCS), Nancy, France
} 
This given, each Parking Function is assigned the "weight"

$$
w(P F)=t^{\operatorname{area}(P F)} q^{\operatorname{dinv}(P F)} Q_{i d e s(P F)}[X]
$$

where

$$
\begin{gathered}
\operatorname{area}(P F)=\sum_{i=1}^{n} u_{i}, \\
\operatorname{dinv}(P F)=\sum_{1 \leq i<j \leq n} \chi\left(u_{i}=u_{j} \& v_{i}<v_{j}\right)+\sum_{1 \leq i<j \leq n} \chi\left(u_{i}=u_{j}+1 \& v_{i}>v_{j}\right),
\end{gathered}
$$

and, for a subset $S \subset\{1,2, \cdots, n-1\}, Q_{S}[X]$ denotes Gessel's fundamental quasi-symmetric function.

In the figure below we have a Parking Function as we usually conveniently depict it. The vector $U=$ $\left(u_{1}, u_{2}, \ldots, u_{n}\right)$ is on its left and the vector $V=\left(v_{1}, v_{2}, \ldots, v_{n}\right)$ is on its right. The shaded cells give the "main diagonal" (or 0-diagonal) of $P F$. The numbers in the lattice cells are the "cars". The path along whose vertical steps we have set the cars is the supporting "Dyck path" of $P F$. The components of $U=\left(u_{1}, u_{2}, \ldots, u_{n}\right)$ give the orders of the diagonals containing the cars. Note that reading the cars by diagonals from right to left starting with the highest diagonal gives

$$
\sigma(P F) \quad=\quad 31857624 .
$$

Thus

$$
i d e s(P F)=\{2,4,6,7\} .
$$

It is easily seen that the sum in (1) gives the total number of cells between the supporting Dyck path and the main diagonal. Note that two cars in the same diagonal with the car on the left smaller than the car on the right will contribute a unit to $\operatorname{dinv}(P F)$ called a "primary dinv". Likewise, a car on the left that is bigger than a car on the right with the latter in the adjacent lower diagonal contributes a unit to $\operatorname{dinv}(P F)$ called a "secondary dinv".

$$
P F=\left[\begin{array}{llllllll}
4 & 6 & 8 & 1 & 3 & 2 & 7 & 5 \\
0 & 1 & 2 & 2 & 3 & 0 & 1 & 1
\end{array}\right] \Longleftrightarrow
$$

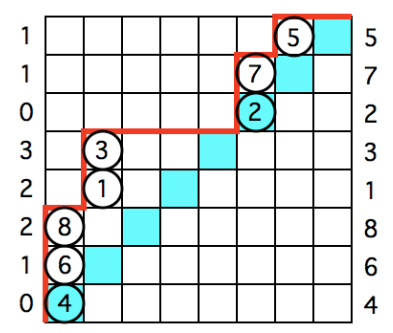

Thus for the Parking Function in (3) we have

$$
\operatorname{area}(P F)=10, \operatorname{dinv}(P F)=4,
$$

which together with (2) give

$$
w(P F)=t^{10} q^{4} Q_{\{2,4,6,7\}}[X] .
$$


In Haglund et al. (2012) Haglund, Morse and Zabrocki introduce an additional statistic, the "diagonal composition" of a Parking function, which we denote by " $p(P F)$." This is the composition whose parts determine the position of the zeros in the vector $U=\left(u_{1}, u_{2}, \ldots, u_{n}\right)$, or equivalently give the lengths of the segments between successive diagonal touches of its Dyck path. For the present example we have

$$
p(P F)=(5,3) \text {. }
$$

Denoting by $\mathcal{P} \mathcal{F}_{n}$ the collection of Parking Functions in the $n \times n$ lattice square, one of the compositional refinements of the Shuffle conjecture due to Haglund-Morse-Zabrocki in Haglund et al. (2012) states that for any composition $p=\left(p_{1}, p_{2}, \ldots, p_{k}\right)$ of $n$ we have

$$
\nabla \mathbf{C}_{p_{1}} \mathbf{C}_{p_{2}} \cdots \mathbf{C}_{p_{k}} 1=\sum_{\substack{P F \in \mathcal{P} \mathcal{F} \\ p(P F)=\left(p_{1}, p_{2}, \ldots, p_{k}\right)}} t^{\operatorname{area}(P F)} q^{\operatorname{dinv}(P F)} Q_{i d e s(P F)}[X]
$$

where " $\nabla$ " is the Bergeron-Garsia operator introduced in [1] and, for each integer $a, \mathbf{C}_{a}$ is the operator plethystically defined by setting for any symmetric function $P[X]$

$$
\mathbf{C}_{a} P[X]=\left.\left(\frac{-1}{q}\right)^{a-1} \sum_{k \geq 0} P\left[X-\frac{1-1 / q}{z}\right] z^{k} h_{k}[X]\right|_{z^{a}} .
$$

Using the device $\theta_{i}$ which acts on the operator $\mathbf{C}_{p}=\mathbf{C}_{p_{1}} \mathbf{C}_{p_{2}} \cdots \mathbf{C}_{p_{k}}$ according to the formula

$$
\theta_{i} \mathbf{C}_{p}=\mathbf{C}_{p-e_{i}}
$$

where $e_{i}$ is the coordinate vector with 1 in the $i^{t h}$ position, we will show that

Theorem 1 For any composition $\left(p_{1}, p_{2}, \ldots, p_{k}\right)$ we have

$$
s_{p_{1}, p_{2}, \ldots, p_{k}}[X]=(-q)^{p_{1}+\cdots+p_{k}-k} \prod_{1 \leq i<j \leq n}\left(1-\theta_{j} / q \theta_{i}\right) \boldsymbol{C}_{p} \boldsymbol{I}
$$

where " $s_{p_{1}, p_{2}, \ldots, p_{k}}[X]$ ” denotes the Schur function indexed by the composition $\left(p_{1}, p_{2}, \ldots, p_{k}\right)$.

To get across the significance of this identity it is best to have a close look at a few special cases. To begin, for $k=2$ with $a \geq b \geq 1, p_{1}=a$ and $p_{2}=b$, (5) becomes

$$
s_{a, b}[X]=(-q)^{a+b-2}\left(1-\theta_{2} / q \theta_{1}\right) \mathbf{C}_{[a, b]} \mathbf{1}=(-q)^{a+b-2}\left(\mathbf{C}_{[a, b]} \mathbf{1}-\mathbf{C}_{[a+1, b-1]} \mathbf{1} / q\right) .
$$

Similarly, for $k=3$ with $a \geq b \geq c \geq 1, p_{1}=a, p_{2}=b$ and $p_{3}=c$, we get

$$
\begin{aligned}
s_{a, b, c}[X]=(-q)^{a+b+c-3}\left(1-\theta_{2} / q \theta_{1}\right)\left(1-\theta_{3} / q \theta_{1}\right)\left(1-\theta_{3} / q \theta_{2}\right) \mathbf{C}_{a} \mathbf{C}_{b} \mathbf{C}_{c} \mathbf{1}= \\
=(-q)^{a+b+c-3\left(\mathbf{C}_{[a, b, c]} \mathbf{1}\right.}-\mathbf{C}_{[a, b+1, c-1]} \mathbf{1} / q-\mathbf{C}_{[a+1, b, c-1]} \mathbf{1} / q-\mathbf{C}_{[a+1, b-1, c]} \mathbf{1} / q \\
+\mathbf{C}_{[a+1, b, c-1]} \mathbf{1} / q^{2}+\mathbf{C}_{[a+1, b+1, c-2]} \mathbf{1} / q^{2} \\
\left.+\mathbf{C}_{[a+2, b-1, c-1]} \mathbf{1} / q^{2}-\mathbf{C}_{[a+2, b, c-2]} \mathbf{1} / q^{3}\right) .
\end{aligned}
$$

These identities suggest that it may be possible to obtain a Parking Function interpretation for $\nabla$ of a Schur function via the compositional refinement of the Shuffle conjecture in (4). 
For example, from (6) we obtain that

$$
\nabla s_{4,3}=q^{4}\left(\nabla \mathbf{C}_{5} \mathbf{C}_{2} \mathbf{1}-q \nabla \mathbf{C}_{4} \mathbf{C}_{3} \mathbf{1}\right) .
$$

From the Haglund-Morse-Zabrocki conjectures, it follows that the sum of the weights of the collection $\Pi[5,2]$ of Parking Functions with diagonal composition $[5,2]$ should be given by the polynomial $\nabla \mathbf{C}_{5} \mathbf{C}_{2}$ and the sum of the weights of the collection $\Pi[4,3]$ of Parking Functions with diagonal composition $[4,3]$ should be given by $\nabla \mathbf{C}_{4} \mathbf{C}_{3}$. This given, to obtain a combinatorial setting for $\nabla s_{4,3}$ it suffices to construct an injection $\phi$ of $\Pi[4,3]$ into $\Pi[5,2]$ that preserves area and ides but increases dinv by one unit, and then identify the complementary collection $\Pi[5,2] \backslash \phi(\Pi[4,3])$ : thereby obtaining the identity

$$
\nabla s_{[4,3]}=q^{4} \sum_{P F \in \Pi[5,2] \backslash(\phi \Pi[4,3])} t^{\operatorname{area}(P F)} q^{\operatorname{dinv}(P F)} Q_{i d e s(P F)}[X]
$$

A look at the identity in (7) suggests that a combinatorial setting for $\nabla s_{a, b, c}$ may be obtained by carrying out an "inclusion-exclusion" process on the collections of Parking Functions with diagonal compositions the indices of the operators occurring in (7).

The task of carrying out the injections yielding such Parking Function settings for the Nabla image of Schur functions is the topic of the author's doctoral thesis which is still in progress. In this article we show how this can be systematically carried out in a variety of examples of Schur functions such as those indexed by two-row or two-column partitions.

We should mention that in Loehr and Warrington (2008) another combinatorial model is conjectured for Nabla Schurs by means of labeled nested Dyck paths. The Loehr-Warrington model stems naturally from the Jacobi-Trudi formula for Schur functions, while the present model stems naturally from Theorem (11) which may be viewed as a $q$-analogue of Jacobi-Trudi. It would make an interesting combinatorial project to see how their model relates to ours, in particular whether their nested labeled Dyck paths can be naturally unraveled into collections of Parking Functions. Even more importantly, if the latter unraveling is carried out, any progress in the resolution of the Loehr-Warrington conjecture may be conducive to significant progress in the resolution of the Haglund-Morse-Zabrocki conjectures.

This writing is divided into three sections. In the first section we give a proof of Theorem (1), in the second section we give some examples in the two part partition cases, in the third and final section we show how the $\mathbf{B}$ operators of Haglund et al. (2012) can be used to give a Parking Function setting to the Nabla image of two-column Schur functions.

\section{A $q$-analogue of the Jacobi-Trudi identity}

In this section it will be convenient to use plethystic notation in dealing with symmetric function identities. A brief introduction to this notational device can be found in the first section of Garsia et al. (2011). Recall that the "row adder" for Schur functions is the operator

$$
\mathbf{S}_{a} P[X]=\left.P\left[X-\frac{1}{z}\right] \Omega[z X]\right|_{z^{a}}
$$

where

$$
\Omega[z X]=\sum_{m \geq 0} z^{m} h_{m}[X]
$$

is the generating function of the homogeneous symmetric functions in the alphabet $X$. 
Proposition 1 For any integral vector $p=\left(p_{1}, p_{2}, \ldots, p_{k}\right)$ we have

$$
s_{p_{1}, p_{2}, \ldots, p_{k}}[X]=\left.\Omega\left[Z_{k} X\right] \prod_{1 \leq i<j \leq k}\left(1-z_{j} / z_{i}\right)\right|_{z_{1}^{p_{1}}, z_{2}^{p_{2}}, \ldots, z_{k}^{p_{k}}}
$$

where $Z_{k}=z_{1}+z_{2}+\cdots+z_{k}$.

\section{Proof:}

It is well known (see Macdonald (1995)) that

$$
s_{p_{1}, p_{2}, \ldots, p_{k}}[X]=\mathbf{S}_{p_{1}} \mathbf{S}_{p_{2}} \cdots \mathbf{S}_{p_{k}} \mathbf{1}
$$

From the definition in $[8$, with $F[X]=\mathbf{1}$, we get,

$$
\begin{aligned}
\mathbf{S}_{p_{1}} \mathbf{S}_{p_{2}} \mathbf{1}=\left.\mathbf{S}_{p_{1}} \Omega\left[z_{2} X\right]\right|_{z_{2}^{p_{2}}}=\left.\Omega\left[z_{2}\left(X-\frac{1}{z_{1}}\right)\right] \Omega\left[z_{1} X\right]\right|_{z_{1}^{p_{1}}, z_{2}^{p_{2}}} \\
\quad=\left.\Omega\left[\left(-z_{2} / z_{1}\right)\right] \Omega\left[z_{1} X+z_{2} X\right]\right|_{z_{1}^{p_{1}}, z_{2}^{p_{2}}}=\left.\left(1-z_{2} / z_{1}\right) \Omega\left[z_{1} X+z_{2} X\right]\right|_{z_{1}^{p_{1}}, z_{2}^{p_{2}}}
\end{aligned}
$$

and by iteration we obtain

$$
\mathbf{S}_{p_{1}} \mathbf{S}_{p_{2}} \cdots \mathbf{S}_{p_{k}} \mathbf{1}=\left.\Omega\left[Z_{k} X\right] \prod_{1 \leq i<j \leq k}\left(1-z_{j} / z_{i}\right)\right|_{z_{1}^{p_{1}}, z_{2}^{p_{2}}, \ldots, z_{k}^{p_{k}}}
$$

Therefore (9) follows from (10).

We are now in a position to give our

\section{Proof of Theorem 1;}

Recall that by definition we have set for any symmetric function $F[X]$

$$
\mathbf{C}_{a} F[X]=\left.\left(-\frac{1}{q}\right)^{a-1} F\left[X-\frac{1-1 / q}{z}\right] \Omega[z X]\right|_{z^{a}} .
$$

Then

$$
\begin{aligned}
(-q)^{p_{1}+p_{2}-2} \mathbf{C}_{p_{1}} \mathbf{C}_{p_{2}} F[X] & =\left.(-q)^{p_{1}-1} \mathbf{C}_{p_{1}} F\left[X-\frac{1-1 / q}{z_{2}}\right] \Omega\left[z_{2} X\right]\right|_{z_{2}^{p_{2}}} \\
& =\left.F\left[X-\frac{1-1 / q}{z_{1}}-\frac{1-1 / q}{z_{2}}\right] \Omega\left[z_{2}\left(X-\frac{1-1 / q}{z_{1}}\right)\right] \Omega\left[z_{1} X\right]\right|_{z_{1}^{p_{1}}, z_{2}^{p_{2}}} \\
& =\left.F\left[X-\frac{1-1 / q}{z_{1}}-\frac{1-1 / q}{z_{2}}\right] \Omega\left[-z_{2} \frac{1-1 / q}{z_{1}}\right] \Omega\left[z_{1} X+z_{2} X\right]\right|_{z_{1}^{p_{1}}, z_{2}^{p_{2}}} .
\end{aligned}
$$

Since $\Omega\left[-z_{2} \frac{1-1 / q}{z_{1}}\right]=\frac{1-z_{2} / z_{1}}{1-z_{2} / q z_{1}}$, we finally have that

$$
(-q)^{p_{1}+p_{2}-2} \mathbf{C}_{p_{1}} \mathbf{C}_{p_{2}} F[X]=\left.F\left[X-\frac{1-1 / q}{z_{1}}-\frac{1-1 / q}{z_{2}}\right] \Omega\left[z_{1} X+z_{2} X\right] \frac{1-z_{2} / z_{1}}{1-z_{2} / q z_{1}}\right|_{z_{1}^{p_{1}}, z_{2}^{p_{2}}} .
$$


By iteration we obtain

$$
(-q)^{p_{1}+\cdots+p_{k}-k} \mathbf{C}_{p_{1}} \cdots \mathbf{C}_{p_{k}} F[X]=\left.F\left[X-\sum_{i=1}^{k} \frac{1-1 / q}{z_{i}}\right] \Omega\left[Z_{k} X\right] \prod_{1 \leq i<j \leq k} \frac{1-z_{j} / z_{i}}{1-z_{j} / q z_{i}}\right|_{z_{1}^{p_{1}}, \ldots, z_{k}^{p_{k}}}
$$

with

$$
Z_{k}=z_{1}+z_{2}+\cdots+z_{k}
$$

Now note that for $F[X]=1$ and for any vector $a=\left(a_{1}, a_{2}, \ldots a_{k}\right)$ we get

$$
\begin{aligned}
& (-q)^{p_{1}-a_{1}+\cdots+p_{k}-a_{k}-k} \mathbf{C}_{p_{1}-a_{1}} \cdots \mathbf{C}_{p_{k}-a_{k}} \mathbf{1}= \\
& =\left.\prod_{1 \leq i<j \leq k} \frac{1-z_{j} / z_{i}}{1-z_{j} / q z_{i}} \Omega\left[X Z_{k}\right]\right|_{z_{1}^{p_{1}-a_{1}} z_{2}^{p_{2}-a_{2}} \cdots z_{k}^{p_{k}-a_{k}}} \\
& =\left.\prod_{1 \leq i<j \leq k} \frac{1-z_{j} / z_{i}}{1-z_{j} / q z_{i}} \Omega\left[X Z_{k}\right] z_{1}^{a_{1}} z_{2}^{a_{2}} \cdots z_{k}^{a_{k}}\right|_{z_{1}^{p_{1}} z_{2}^{p_{2}} \cdots z_{k}^{p_{k}}} .
\end{aligned}
$$

Recalling that the device $\theta_{i}$ acts on the operator $\mathbf{C}_{p}=\mathbf{C}_{p_{1}} \mathbf{C}_{p_{2}} \cdots \mathbf{C}_{p_{k}}$, according to the formula

$$
\theta_{i} \mathbf{C}_{p}=\mathbf{C}_{p-e_{i}}
$$

we can rewrite $[11]$ as

$$
\begin{aligned}
(-q)^{p_{1}+\cdots+p_{k}-k}\left(-\theta_{1} / q\right)^{a_{1}} & \left(-\theta_{2} / q\right)^{a_{2}} \cdots\left(-\theta_{k} / q\right)^{a_{k}} \mathbf{C}_{p} \mathbf{1}= \\
& =\left.\prod_{1 \leq i<j \leq k} \frac{1-z_{j} / z_{i}}{1-z_{j} / q z_{i}} \Omega\left[X Z_{k}\right] z_{1}^{a_{1}} z_{2}^{a_{2}} \cdots z_{k}^{a_{k}}\right|_{z_{1}^{p_{1}} z_{2}^{p_{2} \cdots z_{k}^{p_{k}}}}
\end{aligned}
$$

Thus

$$
(-q)^{p_{1}+\cdots+p_{k}-k} \prod_{1 \leq i<j \leq n}\left(1-\theta_{j} / q \theta_{i}\right) \mathbf{C}_{p} \mathbf{1}=\left.\prod_{1 \leq i<j \leq n}\left(1-z_{j} / z_{i}\right) \Omega\left[X Z_{k}\right]\right|_{z_{1}^{p_{1}} z_{2}^{p_{2} \ldots z_{k}^{p_{k}}}}
$$

This identity combined with (9) gives

$$
(-q)^{p_{1}+\cdots+p_{k}-k} \prod_{1 \leq i<j \leq n}\left(1-\theta_{j} / q \theta_{i}\right) \mathbf{C}_{p} \mathbf{1}=s_{p_{1,2}, \ldots, p_{n}}[X]
$$

as desired. 


\section{The two row Schur function case}

To illustrate the combinatorial reasoning that is needed to give a Parking Function setting to the Nabla image of a two row Schur function we will carry out in full detail the case of $\nabla\left(s_{[n-3,3]}\right)$ which is the simplest non trivial case.

Let $n>5$. Let $\mathcal{N S}_{3}$ denote the collections of Parking Functions with diagonal composition $[n-2,2]$ whose Dyck path terminates according to one of the following three patterns and the cars adjacent to the north steps are required to satisfy the inequalities indicated by the arrows.
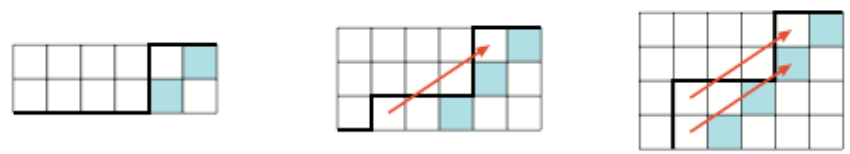

Fig. 1: In the second pattern $v_{n-2}<v_{n}$ and in the third pattern $v_{n-2}<v_{n}$ and $v_{n-3}<v_{n-1}$.

Theorem 2 Assume that the compositional refinement of the Shuffle conjecture in (4) holds. Then,

\section{Proof:}

$$
\nabla(-1)^{n} s_{[n-3,3]}=q^{n-3} \sum_{P F \in \mathcal{N} \mathcal{S}_{3}} t^{\operatorname{area}(P F)} q^{\operatorname{dinv}(P F)} Q_{i d e s(P F)}[X]
$$

We start by constructing an injection $\phi_{3}$ from the collection $\Pi[n-3,3]$ of Parking Functions with diagonal composition $[n-3,3]$ to $\Pi[n-2,2]$, those Parking Functions with diagonal composition $[n-2,2]$. Furthermore, this injection will preserve the area and $i d e s$ of the Parking Functions while increasing the dinv by exactly 1 .

Let $P F$ be a Parking Function with diagonal composition $[n-3,3]$. There are two possible shapes for the rightmost three columns of the Dyck path of $P F$.

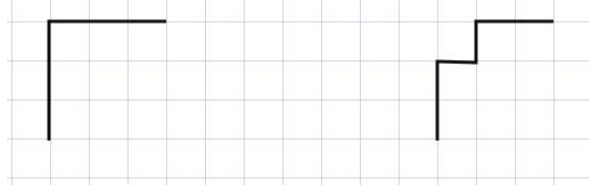

Fig. 2: The rightmost possible columns.

In either shape, since $n>5, P F$ does not hit the diagonal twice in a row. Hence the two steps preceding the last three columns must both be going east.

Between these two shapes, we will have five cases for defining $\phi_{3}(P F)$. For the first, suppose that the last four columns of $P F$ are as in the left side of the figure below. Suppose also that $a<c$. Then replacing the last four columns of $P F$ with the right side of this figure gives a legal Parking Function. Let this be denoted by $P F^{\prime}$

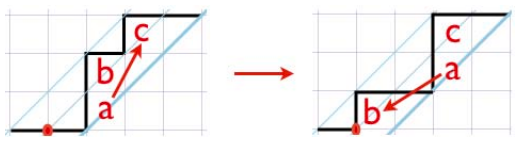

Notice that area $(P F)=$ area $\left(P F^{\prime}\right)$. Furthermore, these two Parking Functions have the same diagonal word and hence $i d e s(P F)=i \operatorname{des}\left(P F^{\prime}\right)$. Notice also that all pairs contributing to the dinv (primary 
or secondary) are unchanged except that the pair $(b, a)$ now also contributes to the secondary dinv. Hence $\operatorname{dinv}\left(P F^{\prime}\right)=\operatorname{dinv}(P F)+1$. Therefore we will set $\phi_{3}(P F)=P F^{\prime}$.

For the second case, suppose that the last four columns of $P F$ are as in the left side of the figure below with $a>c$. Again, let $P F^{\prime}$ be the Parking Function obtained by replacing the last four columns of $P F$ with the columns of the right side of the figure below.

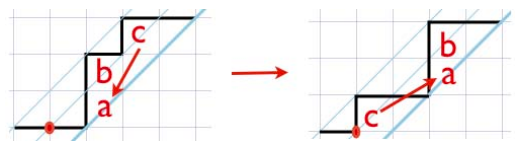

We have that $c<a<b$, so $P F^{\prime}$ is a valid Parking Function. The area is unchanged. However, the diagonal word has changed. In particular, $b$ and $c$ have switched places and we need to show that $i d e s(P F)$ has not been changed. To see this recall that the descent of the inverse of a permutation is the set of all $j$ such that $j+1$ occurs before $j$ in the permutation. This given, the interchange of the order of $c$ and $b$ alters $i d e s(P F)$ only if $c$ and $b$ are consecutive but this is excluded by the inequalities $c<a<b$.

It remains to show that $\operatorname{dinv}\left(P F^{\prime}\right)=\operatorname{dinv}(P F)+1$. But this is true since the pair $(c, b)$ contributes to $\operatorname{dinv}\left(P F^{\prime}\right)$, though it did not contribute to $\operatorname{dinv}(P F)$, and the pair $(c, a)$ is not contributing to the secondary dinv since $c<a$. Hence we can again let $\phi_{3}(P F)=P F^{\prime}$.

We have exhausted the cases corresponding to the right side of Fig. 2. Therefore we will move on to the left side. As we noted before, the two steps preceeding the last three columns must both be east steps. However the step preceeding that could be either north or east.
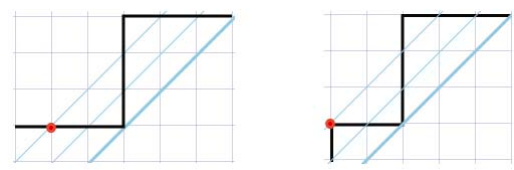

Note that on the right side of the figure above, the step directly before the ones shown must be an east step since $n>5$ and another north step would result in hitting the diagonal.

For the third case, suppose the last three columns of $P F$ are as on the left. Then we can construct $P F^{\prime}$ in the usual way corresponding to the diagram below.

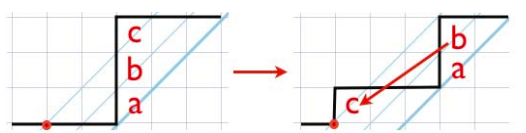

Note $\operatorname{area}(P F)=\operatorname{area}\left(P F^{\prime}\right)$. Also the diagonal word is unchanged for $\operatorname{ides}(P F)=\operatorname{ides}\left(P F^{\prime}\right)$. Furthermore, the only change to the dinv is that $(c, b)$ contributes to the secondary dinv in $P F^{\prime}$. Hence $\operatorname{dinv}\left(P F^{\prime}\right)=\operatorname{dinv}(P F)+1$. Therefore we again set $\phi_{3}(P F)=P F^{\prime}$.

For the fourth case, suppose that the last three columns of $P F$ are as on the left side of the figure below. Suppose also that $c>d$. Let $P F^{\prime}$ be the Parking Function obtained by replacing the last five columns with those on the right side of the figure below.

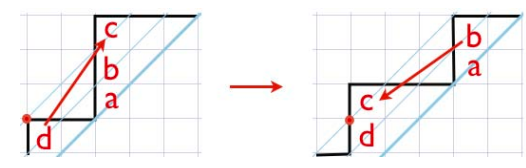


We have $\operatorname{area}(P F)=\operatorname{area}\left(P F^{\prime}\right)$, and $i d e s(P F)=i d e s\left(P F^{\prime}\right)$ since the diagonal word is unchanged. As in the last case, the only change to the dinv being that $(c, b)$ contributes to $\operatorname{dinv}\left(P F^{\prime}\right)$ but not to $\operatorname{dinv}(P F)$. Hence $\phi_{3}(P F)=P F^{\prime}$.

Now for the fifth and final case, suppose that $P F$ is as in the left side of the figure below and $c<d$. Then $a<b<c<d$, so replacing the last 5 columns with those of the right side of the figure gives a Parking Function $P F^{\prime}$

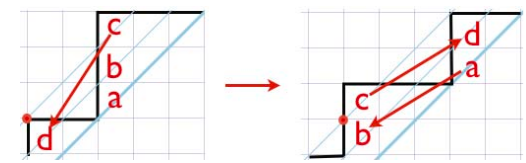

Clearly area $(P F)=\operatorname{area}\left(P F^{\prime}\right)$. The diagonal word has changed however. As in the second case, two labels have switched places in the diagonal word, namely $b$ and $d$. Again, these two labels are not consecutive $(b<c<d)$ so, arguing in just the same way as in the second case, we see that $i d e s(P F)=$ $i d e s\left(P F^{\prime}\right)$. It remains to consider the change to the dinv. Since no labels have been moved to a different diagonal, the only changes to dinv must occur due to pairs of the labels $a, b, c, d$. In $P F$, the pair $(b, d)$ does not contribute to the primary dinv while the pair $(a, d)$ contributes 1 to the secondary $\operatorname{din} v$. In $P F^{\prime}$, the pairs $(b, d)$ and $(b, a)$ contribute to the primary and secondary dinv, respectively, but the pair $(a, d)$ no longer contributes. Hence $\operatorname{dinv}\left(P F^{\prime}\right)=\operatorname{dinv}(P F)+1$ as desired. Therefore we let $\phi_{3}(P F)=P F^{\prime}$.

The following display summarizes the action of $\phi_{3}$ in each of the five cases
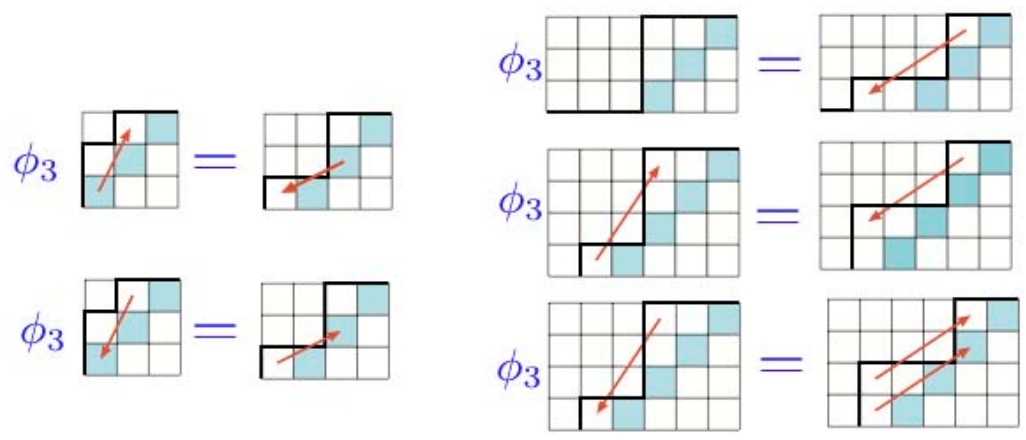

We can thus see that $\phi_{3}$ is an injection $\Pi[n-3,3]$ into $\Pi[n-2,2]$ since the images are disjoint sub collections of $\Pi[n-2,2]$. Moreover note that $\Pi[n-2,2]$ may be partitioned as follows

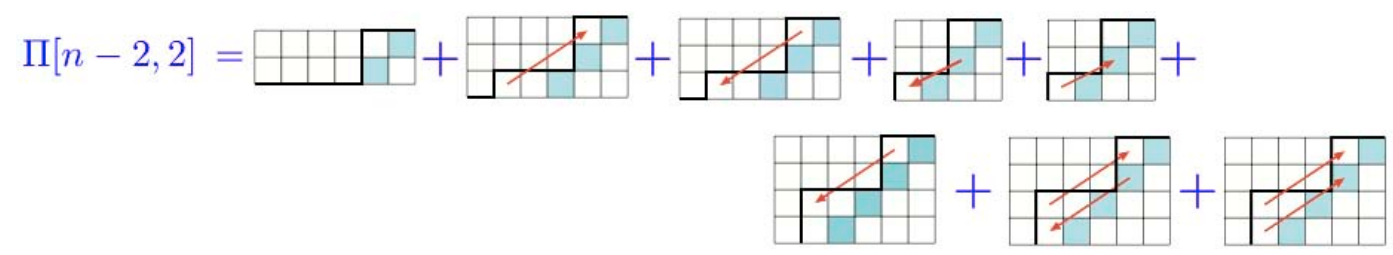

We can thus deduce that

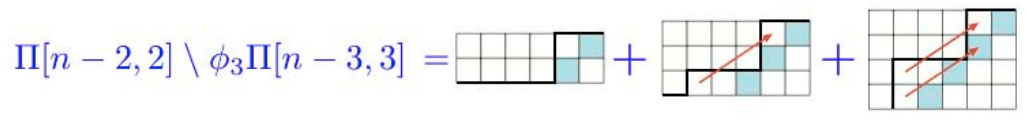


Now by (6), and the Haglund-Morse-Zabrocki conjectures we finally obtain that

$$
\begin{aligned}
(-1)^{n} \nabla\left(s_{[n-3,3]}\right) & =q^{n-3}\left(\sum_{P F \in \Pi[n-2,2]} w(P F)-q \sum_{P F \in \Pi[n-3,3]} w(P F)\right) \\
& =q^{n-3}\left(\sum_{P F \in \Pi[n-2,2]} w(P F)-\sum_{P F \in \Pi[n-3,3]} w\left(\phi_{3}(P F)\right)\right) \\
& =q^{n-3} \sum_{P F \in \mathcal{N} \mathcal{S}_{3}} t^{\operatorname{area}(P F)} q^{\operatorname{dinv}(P F)} Q_{i d e s(P F)}[X] .
\end{aligned}
$$

\section{The Two Column Schur function case}

In the Haglund-Morse-Zabrocki paper Haglund et al. (2012) another Hall-Littlewood type operator "B ${ }_{b}$ " is introduced whose action on a symmetric function $F[X]$ is defined by setting

with

$$
\mathbf{B}_{b}=\omega \widetilde{\mathbf{B}}_{b} \omega
$$

$$
\widetilde{\mathbf{B}}_{b} F[X]=\left.F\left[X-\frac{1-q}{z}\right] \Omega[z X]\right|_{z^{b}}
$$

The significance of these operators in the present context stems from the following identity

Proposition 2 For any integral pair of integers $a>b \geq 1$ we have

$$
s_{2^{b}, 1^{a-b}}[X]=\boldsymbol{B}_{a} \boldsymbol{B}_{b} \boldsymbol{1}-q \boldsymbol{B}_{a+1} \boldsymbol{B}_{b-1} \boldsymbol{I}
$$

Proof: Note that from (13) for $F=\mathbf{1}$ we get

$$
\widetilde{\mathbf{B}}_{b} \mathbf{1}=h_{b}[X]
$$

Thus, again from $(13)$ it follows that

Likewise we get

$$
\widetilde{\mathbf{B}}_{a} \widetilde{\mathbf{B}}_{b} \mathbf{1}=\left.h_{b}\left[X-\frac{1-q}{z}\right] \Omega[z X]\right|_{z^{a}}=\sum_{r=0}^{b} h_{b-r}[X] h_{r}[q-1] h_{r+a}[X]
$$

$$
\widetilde{\mathbf{B}}_{a+1} \widetilde{\mathbf{B}}_{b-1} \mathbf{1}=\sum_{r=0}^{b-1} h_{b-1-r}[X] h_{r}[q-1] h_{r+1+a}[X]=\sum_{r=1}^{b} h_{b-r}[X] h_{r-1}[q-1] h_{r+a}[X]
$$

Now it can easily be shown that we have $h_{r}[q-1]=q^{r}-q^{r-1}$ if $r>0$ and $h_{r}[q-1]=1$ if $r=0$. Thus using these identities we may write

$$
\widetilde{\mathbf{B}}_{a} \widetilde{\mathbf{B}}_{b} \mathbf{1}=h_{b}[X] h_{a}[X]+\sum_{r=1}^{b} h_{b-r}[X]\left(q^{r}-q^{r-1}\right) h_{r+a}[X]
$$

and

$$
q \widetilde{\mathbf{B}}_{a+1} \widetilde{\mathbf{B}}_{b-1} \mathbf{1}=q h_{b-1}[X] h_{1+a}[X]+\sum_{r=2}^{b} h_{b-r}[X]\left(q^{r}-q^{r-1}\right) h_{r+a}[X]
$$


By subtraction we get

$$
\begin{aligned}
\widetilde{\mathbf{B}}_{a} \widetilde{\mathbf{B}}_{b} \mathbf{1}-q \widetilde{\mathbf{B}}_{a+1} \widetilde{\mathbf{B}}_{b-1} \mathbf{1} & =h_{b}[X] h_{a}[X]+h_{b-1}[X](q-1) h_{1+a}[X]-q h_{b-1}[X] h_{1+a}[X] \\
& =h_{b}[X] h_{a}[X]-h_{b-1}[X] h_{1+a}[X]=s_{[a, b]}[X]
\end{aligned}
$$

and since $\omega \mathbf{1}=\mathbf{1}$ from 12 we derive that

$$
\mathbf{B}_{a} \mathbf{B}_{b} \mathbf{1}-q \mathbf{B}_{a+1} \mathbf{B}_{b-1} \mathbf{1}=\omega s_{[a, b]}[X]=s_{2^{b}, 1^{a-b}}[X]
$$

as desired.

In Haglund et al. (2012) it is shown s that the $\mathbf{B}_{b}$ and $\mathbf{C}_{a}$ operators satisfy the commutativity relation

$$
\mathbf{B}_{b} \mathbf{C}_{a}=q \mathbf{C}_{a} \mathbf{B}_{b}
$$

and it is also shown that

$$
\mathbf{B}_{b} \mathbf{1}=\sum_{k=1}^{b} \sum_{\left(p_{1}, p_{2}, \ldots, p_{k}\right) \models b} \mathbf{C}_{p_{1}} \mathbf{C}_{p_{2}} \cdots \mathbf{C}_{p_{k}} \mathbf{1}
$$

By combining (15) with 16 and (14) we can then easily derive that

$$
s_{2^{b}, 1^{a-b}}[X]=\sum_{\alpha \models a} \sum_{\beta \models b} q^{l(\beta)} \mathbf{C}_{\beta} \mathbf{C}_{\alpha} \mathbf{1}-q \sum_{\gamma \models a+1} \sum_{\delta \models b-1} q^{l(\delta)} \mathbf{C}_{\delta} \mathbf{C}_{\gamma} \mathbf{1}
$$

with $l(\beta)$ and $l(\delta)$ denoting the lengths of the compositions $\beta$ and $\delta$ respectively.

The number of summands on the right side of this identity can be further reduced and better organized to facilitate the combinatorial steps needed to obtain the desired Parking function setting for $\nabla s_{2^{b}, 1^{a-b}}[X]$.

To begin by breaking up the sums according as the sizes of the last part of $\beta$ is 1 and first part of $\gamma$ are equal to 1 or not gives

$$
\begin{aligned}
s_{2^{b}, 1^{a-b}}[X]=\sum_{\alpha \models a} \sum_{\tilde{\beta} \models b-1} q^{l(\tilde{\beta})+1} \mathbf{C}_{\tilde{\beta}} \mathbf{C}_{1} \mathbf{C}_{\alpha} \mathbf{1}+\sum_{\alpha \models a} \sum_{\beta \models b ; l \beta_{l(\beta)}>1} q^{l(\beta)} \mathbf{C}_{\beta} \mathbf{C}_{\alpha} \mathbf{1} \\
-\sum_{\tilde{\gamma} \models a} \sum_{\delta \models b-1} q^{l(\delta)+1} \mathbf{C}_{\delta} \mathbf{C}_{1} \mathbf{C}_{\tilde{\gamma}} \mathbf{1}-q \sum_{\gamma \models a+1 ; \gamma_{1}>1} \sum_{\delta \models b-1} q^{l(\delta)} \mathbf{C}_{\delta} \mathbf{C}_{\gamma} \mathbf{1}
\end{aligned}
$$

Canceling the common terms we obtain

$$
s_{2^{b}, 1^{a-b}}[X]=\sum_{\alpha \models a} \sum_{\substack{\beta \models b \\ \beta_{l(\beta)}>1}} q^{l(\beta)} \mathbf{C}_{\beta} \mathbf{C}_{\alpha} \mathbf{1}-q \sum_{\substack{\gamma \models a+1 \\ \gamma_{1}>1}} \sum_{\delta \models b-1} q^{l(\delta)} \mathbf{C}_{\delta} \mathbf{C}_{\gamma} \mathbf{1}
$$

Now splitting once more the sums according to the sizes of the first part of $\alpha$ and the last part of $\beta$ which we will denote $u$ and $v$ respectively, setting $\gamma=u+1, \tilde{\gamma}$ and $\delta=\tilde{\delta}, v-1$ we get

$$
s_{2^{b}, 1^{a-b}}[X]=\sum_{\substack{2 \leq u \leq a \\ 1 \leq v \leq b}}\left(\sum_{\tilde{\alpha} \models a-u} \sum_{\tilde{\beta} \models b-v} q^{l(\tilde{\beta})+1} \mathbf{C}_{\tilde{\beta}} \mathbf{C}_{v} \mathbf{C}_{u} \mathbf{C}_{\tilde{\alpha}} \mathbf{1}-q \sum_{\tilde{\gamma} \models a-u} \sum_{\tilde{\delta} \models b-v} q^{l(\tilde{\delta})+1} \mathbf{C}_{\tilde{\delta}} \mathbf{C}_{v-1} \mathbf{C}_{u+1} \mathbf{C}_{\tilde{\gamma}} \mathbf{1}\right)
$$


This can be rewritten in the form

$$
s_{2^{b}, 1^{a-b}}[X]=\sum_{\substack{2 \leq u \leq a \\ 1 \leq v \leq b}} \sum_{\tilde{\alpha}=a-u} \sum_{\tilde{\beta}=b-v} q^{l(\tilde{\beta})+1} \mathbf{C}_{\tilde{\beta}}\left(\mathbf{C}_{v} \mathbf{C}_{u}-q \mathbf{C}_{v-1} \mathbf{C}_{u+1}\right) \mathbf{C}_{\tilde{\alpha}} \mathbf{1}
$$

and the Haglund-Morse-Zabrocki conjectures give

$$
\nabla s_{2^{b}, 1^{a-b}}[X]=\sum_{\substack{2 \leq u \leq a \\ 1 \leq v \leq b}} \sum_{\tilde{\alpha}=a-u} \sum_{\tilde{\beta} \models b-v} q^{l(\tilde{\beta})+1}(W[\tilde{\beta}, v, u, \tilde{\alpha}]-q W[\tilde{\beta}, v, u, \tilde{\alpha}])
$$

where for convenience we have let $W[\tilde{\beta}, v, u, \tilde{\alpha}]$ and $W[\tilde{\beta}, v, u, \tilde{\alpha}]$ denote the sum of the weight of the collections $\Pi[\tilde{\beta}, v, u, \tilde{\alpha}]$ and $\Pi[\tilde{\beta}, v, u, \tilde{\alpha}]$.

This identity shows that to obtain a Parking Function setting for $\nabla s_{2^{b}, 1^{a-b}}[X]$ we need to construct an injection of $\Pi[\tilde{\beta}, v, u, \tilde{\alpha}]$ into $\Pi[\tilde{\beta}, v, u, \tilde{\alpha}]$ which preserves area and ides and increases dinv by 1 . Now it is not difficult to see that to construct this injection it suffices to be able to carry it out for $\Pi[v, u$,$] into$ $\Pi[v, u]$ and then appropriately transfer the resulting injection to the pairs $\Pi[\tilde{\beta}, v, u, \tilde{\alpha}]$ and $\Pi[\tilde{\beta}, v, u, \tilde{\alpha}]$. and the desired properties will be automatically satisfied as long as all moved cars remain in their diagonal as we have illustrated in the example worked out in section 3.

The general case can be obtained as an inclusion-exclusion of Parking Functions based on the identity

$$
\omega s_{p_{1}, p_{2}, \ldots, p_{k}}=\prod_{1 \leq i \leq j \leq n}\left(1-q \theta_{j} / \theta_{i}\right) \mathbf{B}_{p_{1}} \mathbf{B}_{p_{2}} \ldots \mathbf{B}_{p_{k}} \mathbf{1}
$$

which can be established in a manner analogous to our proof of (5). The realization of this plan is part of the author's ongoing thesis research.

\section{Acknowledgements}

The author is grateful to Angela Hicks for her support and advice during this research.

\section{References}

A. M. Garsia, G. Xin, and M. Zabrocki. Hall-littlewood operators in the theory of parking functions. International Mathematical Research Notices, V. 2011(11), 2011.

J. Haglund, J. Morse, and M. Zabrocki. A compositional refinement of the shuffle conjecture specifying touch points of the dyck path. Canadian J. Math, 64:822-844, 2012.

N. A. Loehr and G. Warrington. Nested quantum dyck paths and $\nabla\left(s_{\lambda}\right)$. International Mathematical Research Notices, Vol 2008, 2008.

I. G. Macdonald. Symmetric functions and Hall polynomials. Oxford Mathematical Monographs, New York, 2 edition, 1995. 